\title{
Estudio de susceptibilidad antimicrobiana de Salmonella en muestras de bovinos lecheros
}

\section{Study of antimicrobial susceptibility of Salmonella in samples of dairy cattle}

\author{
Cruz-Urbano Magaly ${ }^{a}$, José Ignacio Olave-Leyva ${ }^{a}$, Víctor Manuel Martínez-Juárez ${ }^{a, b}$
}

\begin{abstract}
:
The World Organization for Animal Health (OIE) refers to the indiscriminate use of antibiotics has resulted in the emergence of bacterial strains resistant to the mechanisms of action of drugs. Currently, one of the problems in veterinary medicine is antimicrobial resistance that has occurred in several pathogens. Antimicrobials that are commonly used in veterinary medicine; Enrofloxacin, penicillin, cephalosporins among others, have an indiscriminate use in medicine for companion animals and in large species. Due to the public health importance that large species represent for humans, the present work aims to measure antimicrobial resistance specifically of Salmonella spp isolates in dairy cattle. The animals object of the study were Holstein cows with dairy zootechnical function, which is used for the manufacture of cheeses in the production unit studied. Salmonella spp was isolated, which turned out to be multi-resistant to more than 3 antibiotics: ampicillin, carbenicillin, cephalothin, gentamicin and nitrofurantoin.
\end{abstract}

Keywords:

Antibiotics; Salmonella spp; resistance; Public health

Resumen:

La Organización Mundial de Sanidad Animal (OIE) hace referencia a que el uso indiscriminado de antibióticos ha dado como resultado la aparición de cepas bacterianas resistentes a los mecanismos de acción de los fármacos. Actualmente, uno de los problemas en la medicina veterinaria, es la resistencia antimicrobiana que se ha presentado en varios agentes patógenos. Los antimicrobianos que son usados comúnmente en veterinaria; Enrofloxacina, penicilinas, cefalosporinas entre otros, tienen un uso indiscriminado en medicina de animales de compañía y en grandes especies. Debido a la importancia de salud pública que las grandes especies representan para los humanos, el presente trabajo tiene como objetivo medir la resistencia antimicrobiana específicamente de aislados de Salmonella spp en bovinos lecheros. Los animales objeto del estudio fueron vacas Holstein con función zootécnica lechera, misma que se destina para la fabricación de quesos en la unidad de producción estudiada. Se aisló Salmonella spp la cual resultó ser multirresistente a mas de 3 antibióticos: ampicilina, carbenicilina, cefalotina, gentamicina y nitrofurantoína.

Palabras Clave:

Antibióticos; Salmonella spp; resistencia; Salud pública

\section{Introducción}

La organización Mundial de la Salud (OMS) define a las enfermedades transmitidas por alimentos (ETA) como: "El conjunto de síntomas originados por la ingestión de agua o productos alimenticios que contengan agentes biológicos o sustancias tóxicas en cantidades tales que afectan la salud del consumidor en forma aguda o crónica, a nivel individual o de un grupo de personas" 1 .
Las ETA constituyen uno de los problemas de salud más relevantes, tanto en países desarrollados, como en vías de desarrollo. La producción de nuevos alimentos y tecnologías de procesamiento, el uso cada vez más difundido de sistemas centralizados de distribución rápida y el aumento de comercio internacional, representan un desafío tanto para la industria como para los organismos de control 2.

\footnotetext{
${ }^{a}$ Universidad Autónoma del Estado de Hidalgo, Instituto de Ciencias Agropecuarias. Ave. Universidad s/n. Ex Hacienda de Aquetzalpa, Tulancingo, Hidalgo. MÉXICO. Magaly Cruz Urbano, MaggyCu05@outlook.es, https://orcid.org/0000-0002-6819-3553; José Ignacio Olave Leyva, jose_olave6083@uaeh.edu.mx, https://orcid.org/0000-0002-4440-2138; Víctor Manuel Martínez-Juárez, victormj@uaeh.edu.mx, https://orcid.org/00000002-7426-6835

${ }^{\mathrm{b}}$ Autor de Correspondencia, Universidad Autónoma del Estado de Hidalgo, https://orcid.org/0000-0002-7426-6835.
} 
Los cambios de hábitos y tendencias de consumo, la existencia de poblaciones especialmente susceptibles debido al envejecimiento, la desnutrición, personas inmunosuprimidas, niños, mujeres embarazadas y los cambios en las poblaciones microbianas también representan un riesgo desde el punto de vista de las ETA.

En los últimos años se presentaron brotes ocasionados por patógenos emergentes dentro de los cuales destacan: Salmonella en huevos y Salmonella typhimurium en alimentos de origen animal 3.

La salmonelosis es una infección causada por microorganismos que pertenecen a la familia Enterobacteriaceae. Se considera que el género Salmonella consta de dos especies, $S$. entérica y $S$. bongori Los miembros de este género son bacilos gram negativos, no esporulados y anaerobios facultativos. Sus principales huéspedes son el ganado vacuno, aves (pollos y gallinas), porcinos, ovinos, equinos y el humano, y está considerada entre las enfermedades transmitidas por alimentos (ETA), que son causadas por agentes infecciosos específicos como virus, hongos, bacterias y parásitos. Dentro de su sintomatología se encuentran alteraciones gastroentéricas que ponen en riesgo la salud pública 4 .

Además, en la ganadería, esta bacteria es una de las causas principales de enfermedades gastrointestinales en terneros y vacas adultas, se sugiere que podría causar grandes repercusiones en las producciones y en la salud pública, y se tratan con una gran variedad de antimicrobianos, que han sido utilizados a lo largo del tiempo 5. De acuerdo a lo mencionado anteriormente, el objetivo de la presente investigación fue realizar antibiogramas para determinar la resistencia de Salmonella spp., frente a distintos antibióticos usados normalmente en la práctica.

\section{Materiales y métodos}

Las muestran fueron recolectadas en las instalaciones del Rancho Universitario "Lic. Javier Rojo Lugo" de la UAEH, ubicado en el Instituto de Ciencias Agropecuarias en el municipio de Tulancingo perteneciente al Estado de Hidalgo, México, clima templado húmedo, media de $19^{\circ} \mathrm{C}$.

Se tomaron 53 muestras de heces de vacas lecheras del área de producción, se realizó un muestreo aleatorio estratificado de acuerdo con el nivel de producción 22; 27 de alta producción y 26 de baja producción, tomando muestras cada semana de las diferentes vacas durante el periodo de octubre del 2019. Se les realizo un hisopado anal, que fueron sumergidos en tubos de recolección preparados previamente con medio de cultivo líquido de tioglicolato enriquecido ( $5 \mathrm{~mL}$ por tubo), donde se dejaron incubar a temperatura ambiente durante 24 horas antes de ser cultivados 6 .

Las muestras obtenidas fueron cultivadas en un medio de cultivo Agar Salmonella Shigella (SS) (DIBICO ${ }^{\circledR}$ ) el cual es un medio selectivo y de diferenciación para el aislamiento de bacilos entéricos patógenos, en especial los pertenecientes al género Salmonella. Cada semana, se preparó una combinación del medio $(6.5 \mathrm{~g} / 130 \mathrm{~mL}$ de agua destilada $\mathrm{p} / \mathrm{v}$ ), el cual se calentó con agitación constante durante 10 minutos hasta su completa disolución, se esterilizó (condiciones), enfrió y vació en cajas de Petri $(20 \mathrm{~mL})$, previamente esterilizadas. Posteriormente, se sembraron las muestras en el medio de cultivo y se incubaron en condiciones aerobias a $37^{\circ} \mathrm{C}$ durante 24 horas 6 .

La identificación de las bacterias se realizó de acuerdo a las características descritas por [6] con base a las especificaciones de las colonias obtenidas en el cultivo, Incoloro, generalmente con centro de color negro que correspondían al género Salmonella. Los organismos no fermentadores de lactosa forman colonias incoloras. Este último grupo incluye Salmonella. El tiosulfato sódico y el citrato férrico permiten detectar la producción de ácido sulfhídrico, como lo demuestran las colonias con centros de color negro 7 .

De las colonias que se desarrollaron en agar SS, con características macroscópicas que sugieren al género Salmonella, se resembraron en el mismo agar (agar SS), para comprobar que solo se observe crecimiento con un solo tipo de morfología colonial. Después del período de incubación, se obtuvieron (aislaron) 25 colonias bacterianas, a las cuales se les realizaron diferentes pruebas bioquímicas con objeto de determinar y confirmar el género. Dichas pruebas fueron: Rojo de Metilo y Voges Proskauer (MR-VP), SIM, Agar Hierro Tripe azúcar (TSI) y Citrato de Simmons, incubando durante $24-48$ horas a $35^{\circ} \mathrm{C} 6$.

Para medir la resistencia a los antimicrobianos se utilizó la prueba de Kirby Bauer, las bacterias selecciondas se sembraron en medio de cultivo Mueller-Hinton $(20 \mathrm{~mL}$ en cajas Petri estériles), antes de ser incubadas, las bacterias se resuspendieron solución salina al $0.75 \%$ en agitación y se sembraron cubriendo totalmente el medio de cultivo. Se colocaron los antibiogramas en las cajas de Petri y se pusieron a incubar a $35^{\circ} \mathrm{C}$ durante 18 horas 8. Los antibióticos usados fueron: Amikacina $(30 \mathrm{mcg})$, Ampicilina $(10 \mathrm{mcg})$, Carbencilina $(100 \mathrm{mcg})$, Cefalotina 
(10 mcg), Cefotaxima (30 mcg), Ciprofloxacina $(5 \mathrm{mcg})$, Cloranfenicol (30mcg), Gentamicina (10mcg), Netilmicina (30 mcg), Nitrofurantoina $(300 \mathrm{mcg})$, Norfloxacina (10 mcg), Sulfametoxazol / trimetoprim (25mcg), (MULTIBAC ID NEGATIVAS (MULTIDISCOS), los resultados se evaluaron cualitativamente como sensible, intermedio y resistente, donde se evalúa el diámetro del halo de inhibición en un disco y expresando este valor en $\mathrm{mm} 9$.

\section{Resultados y discusión}

De las 53 muestras que se tomaron durante cuatro semanas se aislaron 20 bacterias en el medio SS que presentaban las características pertenecientes al género Salmonella, es decir colonias con centro negro. Posteriormente se hicieron las pruebas bioquímicas y de las muestras aisladas, solo 15 resultaron ser del género Salmonella spp, la presencia de Salmonella se evidenció con una frecuencia del $28 \%$ de las vacas positivas a la prueba. En los antibiogramas realizados, se determinó la resistencia antimicrobiana con los principales fármacos que son utilizados en la ganadería y animales domésticos, los resultados obtenidos mostraron que los aislados de Salmonella spp. resultaron ser resistentes al $100 \%$ respecto a ampicilina, carbenicilina, cefalotina, gentamicina y nitrofurantoína, los 15 aislados de Salmonella spp mostraron ser multirresistente a estos antibióticos, seguido de Amikacina (93\%), cefotaxima (40 $\%)$, siendo ciprofloxacino, cloranfenicol (20 \%) y norfloxacino (13 \%) los que presentaron menor resistencia a los antibióticos probados (Tabla 1). Además, dentro de estos 15 aislados, 4 resultaron ser resistentes a todos los antibióticos probados.

Actualmente, la aparición de bacterias resistentes 0 multirresistentes a fármacos antibacterianos, representa un grave problema de salud pública a nivel mundial; esta problemática requiere una vigilancia constante, situación que desafía a la comunidad científica para la búsqueda de alternativas. La disminución de la eficacia y el aumento de la toxicidad de los antimicrobianos sintéticos agravan el problema; por lo que los científicos están buscando compuestos naturales para obtener soluciones favorables. Los resultados obtenidos en los antibiogramas muestran la multirresistencia de los fármacos que son utilizados con frecuencia en la ganadería, los antibióticos beta lactámicos como ampicilina, cefalotina y carbenicilina, ademas de gentamicina y amikacina (aminoglucósidos).

La enfermedad más común que se observa en los hatos lecheros es la mastitis, la cuál causa grandes perdidas económicas, lo cuál provoca que se utilice la terapia con antimicrobianos y que también son usados como prevención contra enfermedades, acorde a 10, los principales antibióticos usados son del grupo de las penicilinas, cefalosporinas, aminoglucósidos (amikacina, gentamicina) y fluroquinolonas. Dentro de este trabajo resistencia encontrada, en antibióticos como ampicilina, cefalotina y gentamicina coincide al menos con la reportada con estos autores $12,13,14,15,16,17,18$, 19, 20, 21.

En el pasado, en los setentas, los antibióticos empezaron a ser usados como promotores del crecimiento 23 , cabe la posibilidad que esta mayor resistencia encontrada en este trabajo sea debido a ello, a pesar de que en México ya existe un acuerdo donde se eliminan los antibióticos en los alimentos usados en los animales de producción 11, desconociendo si se cumple tal acuerdo, aunado a esto, es probable que el uso indebido y excesivo de antimicrobianos de manera empírica para tratar diversas enfermedades este acelerando el aumento de bacterias resistentes junto con la presencia de Salmonella en el ambiente, particularmente en el agua 4. Además, la multirresistencia se define como la resistencia que presenta la bacteria a 3 o mas antibióticos, en este caso un total de $6(40 \%)$ aislados fueron categorizados como multirresistentes a amikacina, ampicilina, carbenicilina, cefalotina, gentamicina y nitrofurantoína 6, 19, 20.

Tabla 1. Resistencia antimicrobiana en Salmonella aisladas de un hato lechero en producción

\begin{tabular}{lc}
\hline Antibióticos & $\begin{array}{c}\text { Resistencia (\%)/ } \\
\text { Aislados }\end{array}$ \\
\hline Amikacina & $93(14 / 15)$ \\
Ampicilina & $100(15 / 15)$ \\
Carbenicilina & $100(15 / 15)$ \\
Cefalotina & $100(15 / 15)$ \\
Cefotaxima & $40(6 / 15)$ \\
Ciprofloxacino & $20(3 / 15)$ \\
Cloranfenicol & $20(3 / 15)$ \\
Gentamicina & $100(15 / 15)$ \\
Netilmicina & $26(4 / 15)$ \\
Nitrofurantoína & $100(15 / 15)$ \\
Norfloxacino & $13(2 / 15)$ \\
Sulfametoxazol / trimetoprim & $26(4 / 15)$ \\
\hline
\end{tabular}

\section{Conclusiones}

Las bacterias del género Salmonella spp están presentes en bovinos en mayor o menor grado en las unidades lecheras en etapa de producción, ya que estos podrían funcionar como reservorios, sin embargo, es necesario seguir evaluando a largo plazo esta resistencia a los antibióticos y tratar de establecer la resistencia encontrada en Salmonella con los tratamientos utilizados en la unidad de producción. Hay que considerar que esta resistencia encontrada en Salmonella de origen animal 
podría contribuir de manera importante al aumento de resistencia antimicrobiana en patógenos de importancia en salud pública. La utilización de antimicrobianos en las unidades de producción debería de hacerse de manera menos empírica y más basada en la evidencia de conocer que bacterias resistentes podría estar en el hato. Aunado a esto, se deben de mejorar las prácticas de higiene, como lavado de manos, mantenimiento de fuentes de agua limpia para los animales, mantener cubiertos los alimentos para los animales, lo cuál ayudaría a prevenir o disminuir la presencia de Salmonella.

\section{Referencias}

[1] OMS. Farmacorresistencia: Uso de antimicrobianos. Febrero 27, 2019, de OMS Sitio web: https://www.who.int/drugresistance/use/es/

[2] Weiler, N; Orrego, M; Alvarez, M; Huber, C; Ortiz, F; Núñez, Piris, L; Pérez, J. Primeros resultados de la vigilancia integrada de la resistencia antimicrobiana de patógenos transmitidos por alimentos, Campylobacter spp. y Salmonella spp. en tres poblaciones distintas. Paraguay. 2011-2012. Mem. Inst. Investig. Cienc. Salud. 2017. 15(2):64-72.

\section{http://scielo.iics.una.py/pdf/iics/v15n2/1812-9528-iics-15-02-00064.pdf}

[3] Delgado, R. S. Actualidad Ganadera 2019. Obtenido de Actualidad Ganadera: http://www.actualidadganadera.com/articulos/salmonelosisen-vacas-lecheras-en-el-peru.html

[4] Miranda LM, Mondragón AC, Martinez B, Guarddon M, Rodriguez JA. Prevalence and Antimicrobial Resistance Patterns of Salmonella from Different Raw Foods in Mexico. Journal of Food Protection 2009. 72(5): 966-971.

[5] Cabrera-Diaz E, Barbosa-Cardenas CM, Perez-Montaño JA, GonzalezAguilar D, Pacheco-Gallardo C, Barba. J Serotype Diversity, and Antimicrobial Resistance of Salmonella in Ground Beef at Retail Stores in Jalisco State, Mexico. Journal of Food Protection 2013. 76(12):2004-2010.

[6] Markey B, Leonard F, Archambault M, Cullinane A, Maguire D. Clinical Veterinary Microbiology 2013. Londres: Mosby; Edition 2nd Revised ed.

[7] Balouiri M, Sadiki M, Ibnsouda SK. Methods for in vitro evaluating antimicrobial activity: A review. J Pharm Anal, 2015. 6:71-79.

[8] Bauer AW, Kirby WM, Sherris JC, Turck M. Antibiotic susceptibility testing by a standardized single disk method. Am J Clin Pathol. 1966, 45:493-6.

[9] National Committee for Clinical Laboratory Standards. Performance standards for antimicrobial susceptibility testing 2005. 15th informational supplement (M100-S15). National Committee for Clinical Laboratory Standards, Wayne, Pa.

[10] Loo E, Song Lai K, Mansor R. Antimicrobial Usage and Resistance in Dairy Cattle Production. En Veterinary Medicine and Pharmaceuticals 2019. (1-15). Londres: IntechOpen Limited.

[11] Diario Oficial de la Federación (2018). ACUERDO por el que se declara la obligatoriedad de la Estrategia Nacional de Acción contra la Resistencia a los Antimicrobianos. 9 de mayo de 2018. Recuperado de: http://dof.gob.mx/nota_detalle.php?codigo $=5525043 \&$ fecha $=05 \% 2 \mathrm{~F} 06$ \%2F2018

[12] Alexander K, Warnick L, Wiedmann M. Antimicrobial resistant Salmonella in dairy cattle in the United States. Vet Res Commun 2009. 33:191-209

[13] Quesada A, Reginatto G, Ruiz Español A, Colantonio L, Borrone, M. Resistencia antimicrobiana de Salmonella spp aislada de alimentos de origen animal para consumo humano. Rev Peru Med Exp Salud Pública. 2016. 33(1):32-44.

[14] Fuenmayor Y, Rodass-González A, Carruyo G, Hoet A, Wittum T, Narváez-Bravo C. Salmonella Prevalence and Antimicrobial Drug Resistance in Dual-Purpose Cattle Operations in the Eastern Region of Zulia State, Venezuela. Foodborne Pathogens and Disease 2019. Vol. 16, No. 3: 1-9.

[15] Okafor UC, Okafor SC, Ogugua AJ. Occurrence of multidrugresistant Salmonella in cattle carcass and contact surfaces in Kwata slaughterhouse, Awka, Anambra State, Nigeria, Int. J. One Health 2020. 6(1): 49-55.

[16] Delgado-Suárez EJ, Ortíz-López R, Gebreyes WA, Allard MW, Barona-Gómez F, Rubio-Lozano MS. Genomic surveillance links livestock production with the emergence and spread of multi-drug resistant non-typhoidal Salmonella in Mexico. Journal of Microbiology 2019. Vol. 57: 271-280

[17] Ranjbar R, Safapoor Dehkordi F, Heiat M. The Frequency of Resistance Genes in Salmonella enteritidis Strains Isolated from Cattle. Iran J Public Health, Vol. 2020. 49, No.5: 968-974

[18] Chung YH, Kim SY, Chang YH. Prevalence and Antibiotic Susceptibility of Salmonella Isolated from Foods in Korea from 1993 to 2001. Journal of Food Protection 2003. 66 (7):1154-1157.

[19] Davidson KE, Byrne BA, Pires AFA, Magdesian KG, Pereira RV. Antimicrobial resistance trends in fecal Salmonella isolates from northern California dairy cattle admitted to a veterinary teaching hospital, 2002-2016. PLoS ONE 2018. 13(6): e0199928. https://doi.org/10.1371/journal.pone.0199928

[20] Ketema L, Ketema Z, Kiflu B, Alemayehu H, Terefe Y, Ibrahim M, Eguale T. Prevalence and Antimicrobial Susceptibility Profile of Salmonella Serovars Isolated from Slaughtered Cattle in Addis Ababa, Ethiopia. BioMed Research International 2018. Article ID 9794869, 7 pages. https://doi.org/10.1155/2018/9794869

[21] Ayala-Romero C, Ballen-Parada C, Rico-Gaytán M, Chamorro-Tobar I, Zambrano-Moreno D, Poutou-Piñales R, Carrascal-Camacho A. Prevalence of Salmonella spp., in mesenteric pig'sganglia at Colombian benefit plants. Rev. MVZ Córdoba 2018. 23(1):6474-6486.

[22] Torres C, Zarazaga M. Antibióticos como promotores del crecimiento en animales. ¿Vamos por el buen camino? Gac Sanit 2002. 16(2):10912 